\title{
1 Transcriptomic profiling of nematode parasites surviving after vaccine \\ 2 exposure
}

3

4 Guillaume Sallé ${ }^{1,2, *}$, Roz Laing ${ }^{3}$, James A. Cotton ${ }^{2}$, Kirsty Maitland ${ }^{3}$, Axel Martinelli ${ }^{2 \#,}$

$5 \quad$ Nancy Holroyd ${ }^{2}$, Alan Tracey ${ }^{2}$, Matthew Berriman ${ }^{2}$, W. David Smith ${ }^{4}$, George F. J.

6 Newlands $^{4}$, Eve Hanks ${ }^{3}$, Eileen Devaney ${ }^{3}$, Collette Britton ${ }^{3}$

$7 \quad{ }^{1}$ INRA - U. Tours, UMR 1282 ISP Infectiologie et Santé Publique, Centre de recherche Val

8 de Loire, Nouzilly, France

$9{ }^{2}$ Wellcome Trust Sanger Institute, Wellcome Genome Campus, Hinxton, Cambridge, United-

10 Kingdom

$11{ }^{3}$ Institute of Biodiversity, Animal Health and Comparative Medicine, College of Medical,

12 Veterinary and Life Sciences, University of Glasgow, Bearsden Road, Glasgow, United-

13 Kingdom

$14{ }^{4}$ Moredun Research Institute, Pentlands Science Park, Bush Loan, Penicuik, Midlothian,

15 United-Kingdom

16 \#Present address: Global Station for Zoonosis Control, Global Institution for Collaborative

17 Research and Education (GI-CoRE), Hokkaido University, N20 W10 Kita-

$18 \mathrm{ku}$, Sapporo, Japan

$19 *$ Corresponding author

20 Email: gs12@sanger.ac.uk 


\section{Abstract}

23 Some nematode species are economically important parasites of livestock, while others are

24 important human pathogens causing some of the most important neglected tropical diseases.

25 In both humans and animals, anthelmintic drug administration is the main control strategy,

26 but the emergence of drug-resistant worms has stimulated the development of alternative

27 control approaches. Among these, vaccination is considered to be a sustainable and cost

28 effective strategy. Currently, Barbervax ${ }^{\circledR}$ for the ruminant strongylid Haemonchus contortus

29 is the only registered subunit vaccine for a nematode parasite, although a vaccine for the

30 human hookworm Necator americanus is undergoing clinical trials (HOOKVAC

31 consortium). As both these vaccines comprise a limited number of proteins there is potential

32 for selection of nematodes with altered sequence or expression of the vaccine antigens. Here

33 we compared the transcriptome of $H$. contortus populations from sheep vaccinated with

34 Barbervax $^{\circledR}$ with worms from control animals. Barbervax ${ }^{\circledR}$ antigens are native integral

35 membrane proteins isolated from the brush border of the intestinal cells of the adult parasite

36 and many of them are proteases. Our findings provide no evidence for changes in expression

37 of genes encoding Barbervax ${ }^{\circledR}$ antigens in the surviving parasite populations. However,

38 surviving parasites from vaccinated animals showed increased expression of other proteases

39 and regulators of lysosome trafficking, and displayed up-regulated lipid storage and

40 defecation abilities that may have circumvented the vaccine effect. Implications for other

41 potential vaccines for human and veterinary nematodes are discussed. 


\section{Introduction}

45 Gastrointestinal nematodes (GIN) are clinically and economically important parasites of 46 humans (Hotez et al., 2016) and livestock species (Kaplan and Vidyashankar, 2012). Human

47 GIN (e.g. hookworm, roundworm and whipworm) infect over one billion people worldwide,

48 resulting in the loss of over three million disability-adjusted life years (DALYs) in 2013

49 (Hotez et al., 2016). In ruminants, parasitic nematode infections cost the global livestock

50 industry billions of dollars annually in production losses and treatments (Kaplan and

51 Vidyashankar, 2012). GIN therefore impede both human health and wealth and are an 52 aggravating factor of poverty (Rist et al., 2015).

53 Control of veterinary parasites has relied primarily on strategic drug administration

54 (McKellar and Jackson, 2004). However the increase in anthelmintic resistance, particularly

55 multidrug resistance, threatens the viability of the livestock industry in many regions of the 56 world (Kaplan and Vidyashankar, 2012). Similarly, control of human helminthiases involves

57 large-scale community treatment, which has resulted in reductions in GIN prevalence over 58 the last 30 years (Hotez et al., 2016; Clarke et al., 2017). However, there is also the potential 59 for anthelminthic drug failure (Hotez et al., 2016) and recent surveys have indicated a low or variable cure rate of human hookworm infection after benzimidazole treatment, with no reduction in anaemia in some endemic regions (Keiser and Utzinger, 2008;

62 Soukhathammavong et al., 2012).

It is unlikely that novel anthelmintic compounds will be approved at an equivalent 64 pace to the emergence of anthelmintic resistance (Geary et al., 2004). Greater research effort 65 is therefore being directed at vaccine development for more sustainable GIN control in both veterinary and human settings (Hewitson and Maizels, 2014; Hotez et al., 2016). Vaccines

67 may be used alone or combined with drug treatment to reduce the emergence of drug 68 resistance (Lee et al., 2011). In comparison to antimicrobial drugs, there are few examples of 
69 the development of resistance to vaccination in bacterial or viral pathogens (Kennedy and

70 Read, 2017). However, the antigenic complexity and immunoregulatory capacity of

71 nematode parasites makes vaccine development challenging (Hewitson and Maizels, 2014).

72 Only two vaccines are currently commercially available: Barbervax ${ }^{\circledR}$ licensed in Australia in

732014 and comprising native parasite gut membrane glycoproteins of the ovine GIN

74 Haemonchus contortus (Bassetto and Amarante, 2015; Kearney et al., 2016), and Bovilis

75 huskvac $^{\circledR}$, an irradiated larval vaccine for the cattle lungworm Dictyocaulus viviparus

76 (McKeand, 2000). For the human hookworm Necator americanus, a phase 1 clinical vaccine

77 trial has been carried out using recombinant aspartic protease Na-APR-1 combined with

78 gluthatione-S-transferase-1 (Na-GST-1) (Brelsford et al., 2017).

79 Digestion of haemoglobin in haematophagous nematodes like $H$. contortus or

80 hookworms requires activity of different proteolytic enzymes, including aspartic, cysteine

81 and metallo-proteases and exopeptidases (Williamson et al., 2003) underscoring the large

82 expansion of protease gene families identified within the genome of $H$. contortus (Laing et

83 al., 2013; Schwarz et al., 2013). Barbervax $^{\circledR}$ is prepared from gut membrane extracts of $H$.

84 contortus adult worms and contains two major protease fractions, H11 and H-gal-GP (Smith

85 et al., 2001). H11 is a family of microsomal aminopeptidases for which five isoforms have

86 been identified in native extracts (Munn et al., 1997; Roberts et al., 2013), and several related

87 isoforms recently found from genome and transcriptome analysis (Mohandas et al., 2016). H-

88 gal-GP is a 1,000 kDa complex of four zinc metallopeptidases (MEP1-4) and two

89 pepsinogen-like aspartyl proteases (PEP-1 and PEP-2) (Smith et al., 2003), together with

90 additional components (thrombospondin, galectins and cystatin), thought unlikely to be

91 protective (Knox et al., 2003). Vaccination of sheep with either H11 or H-gal-GP

92 individually reduced worm burden and faecal egg count by $70 \%$ and $95 \%$, respectively

93 (Munn et al., 1997; Newton and Munn, 1999; Knox et al., 2003; LeJambre et al., 2008; 
94 Roberts et al., 2013). Cysteine proteases HmCP-1,4 and 6, enriched from adult $H$. contortus

95 gut membrane provided a lower level of protection (Knox et al., 2005). Barbervax ${ }^{\circledR}$ induces

96 circulating antibodies which are ingested by the parasite when it feeds and which inhibit

97 haemoglobinase activity in vitro (Ekoja and Smith, 2010) and probably in vivo. Because the

98 gut-membrane antigens are not exposed to the host immune system during natural infection,

99 Barbervax ${ }^{\circledR}$ relies on the induction of antibodies to "hidden" antigens (Knox et al., 2003).

100 Therefore, it is speculated that the Barbervax ${ }^{\circledR}$ proteins are not under selective pressure

101 during natural infection, but whether vaccine-induced immunity influences levels of gene expression is currently unknown.

The high level of genetic diversity observed in genomic datasets of $H$. contortus

(Laing et al., 2013) and other helminths underpins their capacity for adaptation and

contributes to the evolution of drug resistance (Gilleard and Redman, 2016). It is clear that

pathogens can evolve in response to other interventions, including vaccination, in some cases

leading to vaccine escape and failure (Brueggemann et al., 2007; Kennedy and Read, 2017).

Given the limited number of antigens composing the $H$. contortus vaccine, selection may

arise in the field. Here we compare the transcriptomes of Haemonchus adults surviving in

Barbervax $^{\circledR}$ vaccinated animals with worms recovered from control animals post challenge infection. Identifying any effects that vaccines may have on helminth populations may guide their optimal use in the field.

\section{Materials and methods}

\subsection{Experimental design and collection of parasite material}

116 Adult worms examined in this study were collected on completion of a Barbervax ${ }^{\circledR}$ vaccine 
119 was injected subcutaneously with two doses of Barbervax ${ }^{\circledR}$ four weeks apart, whilst the

120 second, control group was not vaccinated. All sheep were given a challenge infection of

$1215,000 H$. contortus MHco3(ISE) L3 administered per os on the same day as the second

122 vaccination. The MHco3(ISE) strain is susceptible to all broad-spectrum anthelmintics (Roos

123 et al., 2004) and was inbred to produce the material for the $H$. contortus genome sequencing

124 project at the Wellcome Trust Sanger Institute (Laing et al., 2013). All strains were

125 maintained at the Moredun Research Institute. The same H. contortus MHco3(ISE) strain

126 was used to generate the vaccine for this study and to challenge vaccinated and control

127 lambs.

128 Fecal egg counts (FEC) were monitored twice weekly between days 17 and 29 post-challenge

129 by a McMaster technique with a sensitivity of 50 eggs/g. Adult worms were recovered from

130 each sheep at post-mortem 31 days post-challenge. Antibody titres were measured by ELISA,

131 with plates coated with Barbervax ${ }^{\circledR}(50 \mu \mathrm{l}$ per well at $2 \mu \mathrm{g} / \mathrm{ml})$. Serum samples were serially

132 diluted (from $1 / 100$ to $1 / 51200$ ) in PBS/0.5\% Tween and binding detected using mouse anti-

133 sheep IgG (Clone GT-34, Sigma G2904; 1:2500 dilution) and rabbit anti-mouse IgG-HRP

134 conjugate (Dako P0260; 1:1000 dilution). Antibody titres are expressed as the reciprocal of

135 the end-point dilution resulting in an OD of $\geq 0.1$ above the average negative control value.

\subsection{Ethics Statement}

138 Experimental infections were performed at the Moredun Research Institute, UK as described 139 previously (Laing et al., 2013). All experimental procedures were examined and approved by 140 the Moredun Research Institute Experiments and Ethics Committee (MRI E46 11) and were 141 conducted under approved UK Home Office licence (PPL 60/03899) in accordance with the 1421986 Animals (Scientific Procedures) Act. 


\subsection{Extraction protocol, library preparation and sequencing}

145 To avoid any confounding factors from eggs in females or differences in sex ratio between 146 samples, only male worms were used for RNA sequencing. RNA sequencing was carried out 147 on pools of seven to ten surviving $H$. contortus adult worms from each animal. In total, 54 148 worms that survived following challenge infection of the Barbervax vaccinated sheep (V 149 group) and 60 worms from control sheep (C group) were picked for RNA preparations 150 (supplementary table S1).

151 Total RNA was extracted from the worms using a standard Trizol (Thermo Fisher Scientific, 152 15596026) protocol and libraries prepared with the Illumina TruSeq RNA preparation kit 153 before sequencing using a HiSeq 2500 platform with v3 chemistry.

\subsection{Real-time PCR}

Total RNA was extracted from triplicate samples of five female worms from the same populations as the sequenced males. $3 \mu \mathrm{g}$ total RNA was used per oligo(dT) cDNA synthesis

(SuperScript ${ }^{\circledR}$ III First-Strand Synthesis System, ThermoFisher, 18080051) with no-reverse transcriptase controls included for each sample. cDNA was diluted 1:100 for quantitative RTPCR (RT-qPCR) and 1ul added to each reaction. RT-qPCR was carried out following the

Brilliant III Ultra Fast SYBR QPCR Master Mix protocol (Agilent Technologies, 600882) and results analysed using MxPro qPCR Software, Version 4.10. Gene expression was normalised to ama (HCOI01464300) and gpd (HCOI01760600) (Lecova et al., 2015). Primer sequences are listed in Table S2.

\subsection{Improved $\boldsymbol{H}$. contortus assembly and corresponding gene model}

The $H$. contortus MHco3.ISE reference genome assembly used for this study was a snapshot of the latest version as of $14 / 11 / 2014$. This assembly consists of 6,668 scaffolds with a total 
assembly length of $332,877,166 \mathrm{bp}$; of which $22,769,937 \mathrm{bp}$ are sequence gaps. The N50 scaffold length is 5,236,391 bp and N90 length is 30,845 bp. Specifically for this project, preliminary gene models were annotated on this assembly by transferring the gene models from the published (v1.0) genome assembly (Laing et al., 2013) using RATT (Otto et al., 2011) with default parameters, and with a de novo approach using Augustus v2.6.1 (Stanke et al., 2004) with exon boundary 'hints' from the RNAseq data described previously (Laing et al., 2013), mapped against the new reference genome in the same way as in this previous paper.

\subsection{RNAseq data handling and differential expression analysis}

RNAseq data were mapped onto the reference genome using a gene index built with Bowtie2 (Langmead and Salzberg, 2012) and TopHat v2.1.0 (Trapnell et al., 2009) with maximal intron length of $50 \mathrm{Kbp}$ and an inner mate distance of $30 \mathrm{bp}$ that identified $48.8 \%$ of the reads being mapped unambiguously to a gene feature. Counts of reads spanning annotated gene features were subsequently determined with HTSeq v0.6.0 (Anders et al., 2015).

To ensure our biological conclusions are not sensitive to details of the statistical methods used, we implemented two different analysis frameworks for the RNA-seq count data, using the DESeq2 v1.12.4 framework (Love et al., 2014) and the voom function as implemented in the LIMMA package v3.28.21 (Law et al., 2014) in R v3.3.1 (R Core Team, 2016). Genes found to be significantly differentially expressed (DE, adjusted p-value $<5 \%$ ) by both VOOM and DESeq2 analyses were retained. A gene ontology enrichment analysis was performed using the TopGO package v2.26.0 (Alexa, 2016).

Gene identifiers of the vaccine core components, namely MEP-3 (Smith et al., 2000), MEP1,2,4, PEP-1 (Britton et al., 1999) and PEP-2 (Smith et al., 2003) as well as H11, were retrieved via a BLAST search of their nucleotide sequence against the $H$. contortus 
194 MHco3.ISE reference assembly (Laing et al., 2013) in WormBase ParaSite (Howe et al.,

195 2016). The expression levels of candidate housekeeping genes (Lecova et al., 2015) were also

196 retrieved using the gene identifiers associated with their GenBank records (Table 1).

\section{Results}

\subsection{Vaccination greatly reduces faecal egg counts in vaccinated sheep}

Parasitological data confirmed a significant reduction in $H$. contortus infection following

Barbervax vaccination. Over the course of the trial, vaccinated sheep (Group V) shed significantly fewer eggs (mean $390 \pm 639$ eggs per gram faeces (epg), Fig 1A, Table S1) than the control group (Group C) given the same challenge infection dose without prior vaccination (mean 5,914 $\pm 2,628$ epg), representing a 15-fold decrease (Wilcoxon test, $\mathrm{p}=$ 0.002). Vaccinated sheep contained fewer worms, indicated by the significantly lower worm volume collected at necropsy compared to control sheep $(2.8 \mathrm{~mL} \pm 1.9$ versus $6.7 \mathrm{~mL} \pm 3.5$;

Table S1). Among the V group, V_5 showed an outlying egg excretion over the course of the trial (1,647 epg at necropsy; upper 95\% confidence interval limit of 861 epg estimated after 1,000 bootstraps), suggesting a relatively suboptimal vaccine response in this animal. This is supported by the lower antibody titre of this sheep, relative to the other Barbervax vaccinated animals, at day 28 post-challenge infection (Fig 1B).

\subsection{Transcriptional response of worms to host vaccination is dominated by higher} expression of proteases and protease inhibitors

We investigated any changes in $H$. contortus gene expression in worms surviving in vaccinated sheep relative to those surviving in controls. On average $11 \mathrm{M}$ (standard deviation of $1.79 \mathrm{M}$ ) reads were available for each library (Table S1). In PCA of the normalized RNA- 
219 resolved along the $1^{\text {st }}$ axis that separated the experimental groups (Fig S1). Two pools of

220 worms sampled from control sheep, C_4 and C_6, showed atypical behaviour that was

221 resolved along the $2^{\text {nd }}$ PCA axis (Fig S1). These samples were discarded from the dataset for

222 subsequent analyses, resulting in a comparison of $6 \mathrm{~V}$ samples and $4 \mathrm{C}$ samples.

223 We found 52 genes significantly differentially expressed (DE; adjusted p-value $<0.05$ )

224 between the two experimental groups, with six genes exhibiting a fold change above 4, and

22534 genes showing a fold change above 2 (Figures 2 and S2, Table 1 and S3). Adult worm

226 survival following vaccination was associated with an increase in expression of most of the

227 DE genes, i.e. 46 out of 52. Among the top six DE genes, the only down-regulated gene was

228 a glycoside hydrolase domain-containing protein (HCOI00569100, Table 1, Figure 2A).

229 Three of the most highly up-regulated genes encoded proteins containing peptidase domains

230 (HCOI01945600, HCOI01283800, Table 1, Figure 2A), or a peptidase inhibitor I4 domain

231 (HCOI01549900, Table 1, Figure 2A), while two genes were unannotated (HCOI01623600,

232 HCOI01736400). Noticeably, orthologs of HCOI01736400 in D. viviparus

233 (nDv.1.0.1.g04423) or A. caninum (ANCCAN_06626 and ANCCAN_06627) also encoded

234 cathepsin B (cysteine peptidase). Expression of the peptidases (HCOI01945600,

235 HCOI01283800) and HCOI01736400 was validated by quantitative RT-PCR in female

236 worms from the same population as the sequenced males, and confirmed a two to three-fold

237 greater expression of each mRNA in worms surviving in vaccinated sheep compared to

238 controls (Figure 2B).

239 Most of the top six DE genes generally exhibited low transcript counts in control C

240 populations (Table S4), suggesting that their higher expression in worms from group V may

241 be triggered or selected for by the vaccine exposure. Interestingly, 14 genes among the $52 \mathrm{DE}$

242 gene set encoded peptidases or peptidase inhibitors exemplified by the significant enrichment

243 for peptidase activity $\left(p=6.7 \times 10^{-15}\right)$, serine-type $\left(p=9.6 \times 10^{-8}\right)$ and cysteine-type peptidase 
$244\left(p=2.8 \times 10^{-10}\right)$ GO terms (Table S5). This shift toward peptidase activity is also consistent

245 with down-regulation of the gamma interferon-inducible lysosomal thiol reductase (GILT,

246 HCOI02049600, Table S3), which is known to catalyse the reduction of cysteine proteases.

247 Higher expression of two genes involved in the anti-microbial response, the Lys-8 encoding 248 gene (HCOI00041100) associated with lysozyme formation, and the anti-microbial peptide 249 theromacin coding gene (HCOI00456500), was also found in worms surviving in vaccinated 250 animals. A proteinase inhibitor (HCOI01591500) and a prolyl-carboxypeptidase encoding 251 gene (HCOI01624100) showing 99.6\% similarity with contortin 2 (Genbank CAM84574.1, 252 BLASTP, e-value=0) also showed significantly greater expression in the V group (Table S3).

\subsection{Vaccine antigen coding genes are not differentially expressed between experimental groups}

256 Importantly we found that most of the genes encoding the core components of the 257 Barbervax ${ }^{\circledR}$ vaccine (MEPs, PEPs, Aminopeptidases) were not significantly differentially 258 expressed between $\mathrm{V}$ and $\mathrm{C}$ worms or where significant, showed slight over-expression in the 259 V worm population (Table 1, Figure 3). 


\section{Discussion}

263 In comparison to the development of drug resistance, vaccine resistance has rarely been 264 reported in viruses or bacteria (Kennedy and Read, 2017). These contrasting findings may

265 relate both to the prophylactic use of vaccines, which prevent the spread of resistant mutants

266 among hosts, and the multiplicity of pathways targeted by the host immune response

267 following vaccination (Kennedy and Read, 2017). However, highly diverse populations, such

268 as H. contortus (Gilleard and Redman, 2016) likely encompass a wide range of genotypes

269 that could be differentially selected, ultimately leading to vaccine resistance through

270 replacement (Martcheva et al., 2008; Weinberger et al., 2011; Barnett et al., 2015).

271 Resistance to all but the newest anthelmintic drugs is common and widespread amongst

272 gastrointestinal nematode parasites of ruminants. Barbervax ${ }^{\circledR}$, which is specific for $H$.

273 contortus, is the only vaccine registered for a gut dwelling nematode of any host. While this

274 vaccine provides a useful level of protection mediated mainly by reducing pasture

275 contamination, a small proportion of worms do survive vaccination. Here, we investigated

276 whether the transcriptome of these survivors differed from those of control worms.

277 In order to generate enough genetic material for sequencing and to avoid any contamination

278 by egg-specific transcripts, this study focused on male worms only. Consequently, our

279 experiment could not resolve the observed sex-specific effect of the Barbervax ${ }^{\circledR}$ vaccine, i.e.

280 the vaccine being more efficient on females than males (Smith and Smith, 1993), although

281 we were able to confirm some of the observed transcriptional differences in female worms

282 recovered from the same animals. Our data shed light on transcription modifications involved

283 in the survival of male worms and provided insights into the mechanisms associated with

284 their survival following vaccination.

285 Since both experimental groups exhibited similar levels of vaccine antigen transcripts, there

286 was no evidence for increased expression of vaccine targets which could mediate vaccine 
287 survival. However a metallopeptidase and an exopeptidase, belonging to the same functional

288 families (Rawlings et al., 2010) as the vaccine MEP (M13 peptidase) and H11 (M1

289 peptidase) respectively, were over-expressed in the vaccine survivors although it is not clear

290 whether these could compensate for vaccine peptidases. Instead, survival following

291 Barbervax $^{\circledR}$ vaccination was associated with enhanced expression of a limited subset of

292 genes, mainly encoding cysteine peptidases. Differential tuning of a GILT-like gene, i.e.

293 down-regulated in worms surviving the vaccine response, would also support proteolytic

294 function as an important feature for vaccine survival, as this pleiotropic gene is known to

295 modulate cysteine protease activity and stability (Rausch and Hastings, 2015). In addition,

296 there was an indication of higher selection pressure on a lyst-1 orthologue, a regulator of

297 endosomal trafficking in C. elegans polarized epithelial cells (de Souza et al., 2007), that may

298 share the same function in $H$. contortus and thus contribute to efficient processing of protein

299 material from the intestinal lumen. This suggests that regulation of the proteolytic pathways

300 in vaccine survivors may result in improved survival. While the precise function of cysteine

301 peptidases is hard to infer in silico, current knowledge from in vitro studies points to their

302 role in the proteolytic cascade responsible for degrading haemoglobin or immunoglobulin $\mathrm{G}$

303 (Williamson et al., 2003). Perhaps worms that over-express these proteins may either

304 maintain blood coagulation and digestion, or are able to degrade host IgG stimulated by the

305 vaccine challenge (Munn et al., 1997; Ekoja and Smith, 2010) to evade the vaccine response,

306 or some combination of both. Indeed the vaccine is proposed to disrupt digestion in the worm

307 gut by blocking the function of the intestinal proteases it targets. Processing of ingested

308 proteins by an alternative proteolytic pathway may improve the survival and/or fecundity of

309 worms suffering dietary restriction. In addition, the over-expression of a myo-inositol-1

310 phosphate synthase in vaccine survivors may also support this theory as this gene is known to 
311 act on lipid storage (Ashrafi et al., 2003) and in the defecation cycle (Tokuoka et al., 2008),

312 both critical in the digestion process, and hence impacting worm growth and lifespan.

313 Interestingly, the most highly differentially expressed genes show a low level of expression

314 in worms from the control group, suggesting that the vaccine response may have induced

315 their overexpression in the vaccine survivors or alternatively, that the vaccine selects for

316 natural variation in expression of these genes. Additional transcriptomic evaluation of the

317 offspring of each worm subpopulation, before and after vaccine exposure, would help

318 confirm this observation and distinguish between a regulatory response to vaccine-induced

319 immunity and genetic differences influencing gene expression.

320 Whilst this study focuses on a species of veterinary significance, our findings may have

321 relevance to other species. Indeed our results suggest that $H$. contortus may be able to

322 compensate for vaccine-mediated immunity after vaccine exposure and a similar situation

323 may apply in other parasitic nematode systems.

\section{Conclusions}

326 Our data suggest that parasite populations surviving Barbervax ${ }^{\circledR}$ immunisation are able to 327 optimize their proteolytic machinery, involving both peptidases and regulators of lysosome 328 trafficking, and display better lipid storage and/or defecation abilities which may enhance 329 survival in the face of a robust vaccine-induced immune response. While our experiment was 330 not designed to detect genetic selection to the vaccine response, an "evolve and 331 resequencing" approach to contrast changes in allele frequencies in vaccinated and 332 unvaccinated populations through time, across multiple generations of vaccine challenge, 333 could help resolve the potential for adaptation following vaccination. 


\section{Acknowledgements}

335 We thank Stephen Doyle for advice and comments on the manuscript and the biological

336 services staff at MRI for their expert animal care. JAC, NH, AM, AT and MB are supported

337 by the Wellcome Trust via their core funding of the Wellcome Trust Sanger Institute (grant

338 206194). JAC, NH, AT, MB, KM, RL, ED and CB are supported by BBSRC grant

$339 \mathrm{BB} / \mathrm{M} 003949 / 1$ (BUG), GS has received the support of the EU in the framework of the

340 Marie-Curie FP7 COFUND People Programme, through the award of an AgreenSkills (grant

agreement $n^{\circ}$ 267196) and AgreenSkills+ fellowships (grant agreement $n^{\circ} 609398$ ). The

funders had no role in study design, data collection and analysis, decision to publish, or

preparation of the manuscript.

\section{References}

Alexa, A., Rahnenfuhrer, J., 2016. topGO: Enrichment Analysis for Gene Ontology.

Anders, S., Pyl, P.T., Huber, W., 2015. HTSeq--a Python framework to work with high-throughput sequencing data. Bioinformatics 31, 166-169.

Ashrafi, K., Chang, F.Y., Watts, J.L., Fraser, A.G., Kamath, R.S., Ahringer, J., Ruvkun, G., 2003. Genome-wide RNAi analysis of Caenorhabditis elegans fat regulatory genes. Nature 421, 268-272.

Barnett, T.C., Lim, J.Y., Soderholm, A.T., Rivera-Hernandez, T., West, N.P., Walker, M.J., 2015. Hostpathogen interaction during bacterial vaccination. Curr Opin Immunol 36, 1-7.

Bassetto, C.C., Amarante, A.F., 2015. Vaccination of sheep and cattle against haemonchosis. J Helminthol 89, 517-525.

Brelsford, J.B., Plieskatt, J.L., Yakovleva, A., Jariwala, A., Keegan, B.P., Peng, J., Xia, P., Li, G., Campbell, D., Periago, M.V., Correa-Oliveira, R., Bottazzi, M.E., Hotez, P.J., Diemert, D., Bethony, J.M., 2017. Advances in neglected tropical disease vaccines: Developing relative potency and functional assays for the Na-GST-1/Alhydrogel hookworm vaccine. PLoS Negl Trop Dis 11, e0005385.

Britton, C., Redmond, D.L., Knox, D.P., McKerrow, J.H., Barry, J.D., 1999. Identification of promoter elements of parasite nematode genes in transgenic Caenorhabditis elegans. Mol Biochem Parasitol 103, 171-181.

Brueggemann, A.B., Pai, R., Crook, D.W., Beall, B., 2007. Vaccine escape recombinants emerge after pneumococcal vaccination in the United States. PLoS Pathog 3, e168.

Clarke, N.E., Clements, A.C., Doi, S.A., Wang, D., Campbell, S.J., Gray, D., Nery, S.V., 2017. Differential effect of mass deworming and targeted deworming for soil-transmitted helminth control in children: a systematic review and meta-analysis. Lancet 389, 287-297.

de Souza, N., Vallier, L.G., Fares, H., Greenwald, I., 2007. SEL-2, the C. elegans neurobeachin/LRBA homolog, is a negative regulator of lin-12/Notch activity and affects endosomal traffic in polarized epithelial cells. Development 134, 691-702. 
Ekoja, S.E., Smith, W.D., 2010. Antibodies from sheep immunized against Haemonchus contortus with $\mathrm{H}$-gal-GP inhibit the haemoglobinase activity of this protease complex. Parasite Immunol 32 , 731-738.

Geary, T.G., Conder, G.A., Bishop, B., 2004. The changing landscape of antiparasitic drug discovery for veterinary medicine. Trends Parasitol 20, 449-455.

Gilleard, J.S., Redman, E., 2016. Genetic Diversity and Population Structure of Haemonchus contortus. Adv Parasitol 93, 31-68.

Hewitson, J.P., Maizels, R.M., 2014. Vaccination against helminth parasite infections. Expert Rev Vaccines 13, 473-487.

Hotez, P.J., Strych, U., Lustigman, S., Bottazzi, M.E., 2016. Human anthelminthic vaccines: Rationale and challenges. Vaccine 34, 3549-3555.

Howe, K.L., Bolt, B.J., Shafie, M., Kersey, P., Berriman, M., 2016. WormBase ParaSite - a comprehensive resource for helminth genomics. Mol Biochem Parasitol.

Kaplan, R.M., Vidyashankar, A.N., 2012. An inconvenient truth: Global worming and anthelmintic resistance. Veterinary Parasitology 186, 70-78.

Kearney, P.E., Murray, P.J., Hoy, J.M., Hohenhaus, M., Kotze, A., 2016. The 'Toolbox' of strategies for managing Haemonchus contortus in goats: What's in and what's out. Vet Parasitol 220, 93-107.

Keiser, J., Utzinger, J., 2008. Efficacy of current drugs against soil-transmitted helminth infections: systematic review and meta-analysis. JAMA 299, 1937-1948.

Kennedy, D.A., Read, A.F., 2017. Why does drug resistance readily evolve but vaccine resistance does not? Proc Biol Sci 284.

Knox, D.P., Redmond, D.L., Newlands, G.F., Skuce, P.J., Pettit, D., Smith, W.D., 2003. The nature and prospects for gut membrane proteins as vaccine candidates for Haemonchus contortus and other ruminant trichostrongyloids. Int J Parasitol 33, 1129-1137.

Knox, D.P., Smith, S.K., Redmond, D.L., Smith, W.D., 2005. Protection induced by vaccinating sheep with a thiol-binding extract of Haemonchus contortus membranes is associated with its protease components. Parasite Immunol 27, 121-126.

Laing, R., Kikuchi, T., Martinelli, A., Tsai, I.J., Beech, R.N., Redman, E., Holroyd, N., Bartley, D.J., Beasley, H., Britton, C., Curran, D., Devaney, E., Gilabert, A., Hunt, M., Jackson, F., Johnston, S.L., Kryukov, I., Li, K., Morrison, A.A., Reid, A.J., Sargison, N., Saunders, G.I., Wasmuth, J.D., Wolstenholme, A., Berriman, M., Gilleard, J.S., Cotton, J.A., 2013. The genome and transcriptome of Haemonchus contortus, a key model parasite for drug and vaccine discovery. Genome Biol 14, R88. Langmead, B., Salzberg, S.L., 2012. Fast gapped-read alignment with Bowtie 2. Nat Methods 9, 357359.

Law, C.W., Chen, Y., Shi, W., Smyth, G.K., 2014. voom: Precision weights unlock linear model analysis tools for RNA-seq read counts. Genome Biol 15, R29.

Lecova, L., Ruzickova, M., Laing, R., Vogel, H., Szotakova, B., Prchal, L., Lamka, J., Vokral, I., Skalova, L., Matouskova, P., 2015. Reliable reference gene selection for quantitative real time PCR in Haemonchus contortus. Mol Biochem Parasitol 201, 123-127.

Lee, B.Y., Bacon, K.M., Bailey, R., Wiringa, A.E., Smith, K.J., 2011. The potential economic value of a hookworm vaccine. Vaccine 29, 1201-1210.

LeJambre, L.F., Windon, R.G., Smith, W.D., 2008. Vaccination against Haemonchus contortus: performance of native parasite gut membrane glycoproteins in Merino lambs grazing contaminated pasture. Vet Parasitol 153, 302-312.

Love, M.I., Huber, W., Anders, S., 2014. Moderated estimation of fold change and dispersion for RNA-seq data with DESeq2. Genome Biol 15, 550.

Martcheva, M., Bolker, B.M., Holt, R.D., 2008. Vaccine-induced pathogen strain replacement: what are the mechanisms? J R Soc Interface 5, 3-13.

McKeand, J.B., 2000. Vaccine development and diagnostics of Dictyocaulus viviparus. Parasitology 120 Suppl, S17-23. 
420

421

422

423

424

425

426

427

428

429

430

431

432

433

434

435

436

437

438

439

440

441

442

443

444

445

446

447

448

449

450

451

452

453

454

455

456

457

458

459

460

461

462

463

464

465

466

467

468

469

470
McKellar, Q.A., Jackson, F., 2004. Veterinary anthelmintics: old and new. Trends Parasitol 20, 456461.

Mohandas, N., Young, N.D., Jabbar, A., Korhonen, P.K., Koehler, A.V., Hall, R.S., Hu, M., Hofmann, A., Gasser, R.B., 2016. The complement of family M1 aminopeptidases of Haemonchus contortus-Biotechnological implications. Biotechnol Adv 34, 65-76.

Munn, E.A., Smith, T.S., Smith, H., James, F.M., Smith, F.C., Andrews, S.J., 1997. Vaccination against Haemonchus contortus with denatured forms of the protective antigen H11. Parasite Immunol 19, 243-248.

Newton, S.E., Munn, E.A., 1999. The development of vaccines against gastrointestinal nematode parasites, particularly Haemonchus contortus. Parasitol Today 15, 116-122.

Otto, T.D., Dillon, G.P., Degrave, W.S., Berriman, M., 2011. RATT: Rapid Annotation Transfer Tool. Nucleic Acids Res 39, e57.

R Core Team, 2016. R: A Language and Environment for Statistical Computing. R Foundation for Statistical Computing, Vienna.

Rausch, M.P., Hastings, K.T., 2015. Diverse cellular and organismal functions of the lysosomal thiol reductase GILT. Mol Immunol 68, 124-128.

Rawlings, N.D., Barrett, A.J., Bateman, A., 2010. MEROPS: the peptidase database. Nucleic Acids Res 38, D227-233.

Rist, C.L., Garchitorena, A., Ngonghala, C.N., Gillespie, T.R., Bonds, M.H., 2015. The Burden of Livestock Parasites on the Poor. Trends Parasitol 31, 527-530.

Roberts, B., Antonopoulos, A., Haslam, S.M., Dicker, A.J., McNeilly, T.N., Johnston, S.L., Dell, A., Knox, D.P., Britton, C., 2013. Novel expression of Haemonchus contortus vaccine candidate aminopeptidase $\mathrm{H} 11$ using the free-living nematode Caenorhabditis elegans. Vet Res 44, 111.

Roos, M.H., Otsen, M., Hoekstra, R., Veenstra, J.G., Lenstra, J.A., 2004. Genetic analysis of inbreeding of two strains of the parasitic nematode Haemonchus contortus. Int J Parasitol 34, 109-115.

Schwarz, E.M., Korhonen, P.K., Campbell, B.E., Young, N.D., Jex, A.R., Jabbar, A., Hall, R.S., Mondal, A., Howe, A.C., Pell, J., Hofmann, A., Boag, P.R., Zhu, X.Q., Gregory, T., Loukas, A., Williams, B.A., Antoshechkin, I., Brown, C., Sternberg, P.W., Gasser, R.B., 2013. The genome and developmental transcriptome of the strongylid nematode Haemonchus contortus. Genome Biol 14, R89.

Smith, W.D., Pettit, D., Smith, S.K., 2001. Cross-protection studies with gut membrane glycoprolein antigens from Haemonchus contortus and Teladorsagia circumcincta. Parasite Immunology 23, 203211.

Smith, W.D., Skuce, P.J., Newlands, G.F., Smith, S.K., Pettit, D., 2003. Aspartyl proteases from the intestinal brush border of Haemonchus contortus as protective antigens for sheep. Parasite Immunol 25, 521-530.

Smith, W.D., Smith, S.K., 1993. Evaluation of aspects of the protection afforded to sheep immunised with a gut membrane protein of Haemonchus contortus. Res Vet Sci 55, 1-9.

Smith, W.D., Smith, S.K., Pettit, D., Newlands, G.F., Skuce, P.J., 2000. Relative protective properties of three membrane glycoprotein fractions from Haemonchus contortus. Parasite Immunol 22, 63-71. Soukhathammavong, P.A., Sayasone, S., Phongluxa, K., Xayaseng, V., Utzinger, J., Vounatsou, P., Hatz, C., Akkhavong, K., Keiser, J., Odermatt, P., 2012. Low efficacy of single-dose albendazole and mebendazole against hookworm and effect on concomitant helminth infection in Lao PDR. PLoS Negl Trop Dis 6, e1417.

Stanke, M., Steinkamp, R., Waack, S., Morgenstern, B., 2004. AUGUSTUS: a web server for gene finding in eukaryotes. Nucleic Acids Res 32, W309-312.

Tokuoka, S.M., Saiardi, A., Nurrish, S.J., 2008. The mood stabilizer valproate inhibits both inositoland diacylglycerol-signaling pathways in Caenorhabditis elegans. Mol Biol Cell 19, 2241-2250.

Trapnell, C., Pachter, L., Salzberg, S.L., 2009. TopHat: discovering splice junctions with RNA-Seq. Bioinformatics 25, 1105-1111.

Weinberger, D.M., Malley, R., Lipsitch, M., 2011. Serotype replacement in disease after pneumococcal vaccination. Lancet 378, 1962-1973. 
471 Williamson, A.L., Brindley, P.J., Knox, D.P., Hotez, P.J., Loukas, A., 2003. Digestive proteases of blood-

472 feeding nematodes. Trends Parasitol 19, 417-423.

473

474 Figure 1. Faecal egg counts and anti-Barbervax IgG titer of individual sheep

475 Fig 1A Faecal egg counts from each of the 12 sheep in the trial were plotted for each 476 available time point post challenge. The plot shows a 15 -fold difference in egg excretion 477 between vaccinated and control sheep on day 29 post challenge infection. Dots for V__ $1, \mathrm{~V} \_3$ 478 and $V \_4$ overlap around 0 as a result of low counts.

479 Fig 1B Faecal egg count measured at necropsy plotted against respective anti-Barbervax ${ }^{\circledR}$ 480 vaccine $\mathrm{IgG}$ titer, showing a negative correlation between vaccine response and egg count.

Figure 2. Expression level of the top differentially expressed genes within each experimental group (2A) and associated correlation with faecal egg count in control populations (2B)

484 A. A boxplot for all six genes that exhibited an absolute log-transformed fold change of 2 485 between the experimental conditions. Dcp stands for "Domain Containing Protein".

B. Fold change in expression level of selected genes, by qRT-PCR, shown relative to C control population. qRT-PCR was carried out on RNA extracted from adult female worms. HCOI01283800: Peptidase C1A domain containing protein; HCOI01549900: Protease inhibitor I4 domain containing protein; HCOI01736400: ortholog to cathepsin B in $D$. viviparus and A. caninum.

Figure 3. Expression level for the vaccine antigen coding genes

Figure 3 shows the normalized transcript counts for known vaccine antigen coding genes.

494 Each dot stands for the transcript count measured in a pool of worms from vaccinated (V, 495 green dots) or control (C, red dots) sheep. Some of the dots overlap because of similar 496 expression levels. 
bioRxiv preprint doi: https://doi org/10.1101/144980; this version posted October 24, 2017. The copyright holder for this preprint (which was not certified by peer review) is the author/funder, who has granted bioRxiv a license to display the preprint in perpetuity. It is made available under aCC-BY-NC 4.0 International license.

497

498 
Table 1. Gene of interest expression levels, fold change and associated p-values

\begin{tabular}{|c|c|c|c|c|c|c|c|c|c|c|c|}
\hline & Gene ID & $\begin{array}{l}\text { Mean } \\
\text { count }^{\mathrm{a}}\end{array}$ & $\begin{array}{c}\operatorname{logFC} C^{b} \\
\text { DESeq } 2\end{array}$ & $\begin{array}{r}\text { adj. } \mathbf{P}^{c} \\
\text { DESeq } 2\end{array}$ & $\begin{array}{c}\operatorname{logFC} C^{b} \\
\text { VOOM }\end{array}$ & $\begin{array}{r}\text { adj. } P^{\mathbf{c}} \\
\text { VOOM }\end{array}$ & $\begin{array}{r}\text { Correlation } \\
\text { with } \\
\text { FEC29 }^{\mathbf{d}}\end{array}$ & WormBase ParaSite Gene description & $\begin{array}{c}\text { C. elegans } \\
\text { orthologue }\end{array}$ & $\begin{array}{r}\text { Candidate } \\
\text { Gene Name }\end{array}$ & $\begin{array}{r}\text { Genbank Acc. } \\
\text { Number }\end{array}$ \\
\hline \multirow{6}{*}{ 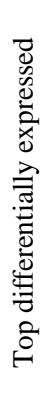 } & HCOI00569100 & 24.21 & -2.39 & $2.40 \mathrm{E}-13$ & -5.16 & 4.55E-03 & $0.63(0.05)$ & $\begin{array}{r}\text { Glycoside hydrolase domain containing } \\
\text { protein [U6P060] }\end{array}$ & $\mathrm{n} / \mathrm{a}$ & $\mathrm{n} / \mathrm{a}$ & $\mathrm{n} / \mathrm{a}$ \\
\hline & HCOI01945600 & 2000.03 & 2.02 & 2.33E-16 & 2.39 & 9.83E-04 & $-0.64(0.05)$ & $\begin{array}{r}\text { Peptidase A1 domain containing protein } \\
\text { [U6PP66] }\end{array}$ & pcl, Bace & $\mathrm{n} / \mathrm{a}$ & $\mathrm{n} / \mathrm{a}$ \\
\hline & HCOI01623600 & 23.12 & 2.03 & 2.05E-09 & 4.21 & 6.77E-03 & $-0.79(0.01)$ & $\mathrm{n} / \mathrm{a}$ & $\mathrm{n} / \mathrm{a}$ & $\mathrm{n} / \mathrm{a}$ & $\mathrm{n} / \mathrm{a}$ \\
\hline & HCOI01283800 & 38840.11 & 2.15 & 3.58E-15 & 2.79 & 1.28E-03 & $-0.76(0.01)$ & $\begin{array}{l}\text { Peptidase C1A domain containing protein } \\
\text { [U6P6R9] }\end{array}$ & CtsB1 & $\mathrm{n} / \mathrm{a}$ & $\mathrm{n} / \mathrm{a}$ \\
\hline & HCOI01549900 & 1104.78 & 2.20 & $6.42 \mathrm{E}-16$ & 2.86 & 1.31E-03 & $-0.73(0.02)$ & $\begin{array}{r}\text { Protease inhibitor I4 domain containing } \\
\text { protein [U6PNP0] }\end{array}$ & $\begin{array}{r}\text { srp- } \\
1,2,3,6,7,8\end{array}$ & $\mathrm{n} / \mathrm{a}$ & n/a ç \\
\hline & HCOI01736400 & 2678.92 & 2.49 & 4.60E-31 & 3.01 & 7.91E-05 & $-0.81(0.004)$ & $\mathrm{n} / \mathrm{a}$ & CtsB1 & $\mathrm{n} / \mathrm{a}$ & $\mathrm{n} / \mathrm{a}$ \\
\hline \multirow{7}{*}{ 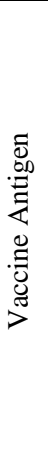 } & HCOI01993300 & 4049.71 & 0.30 & $3.09 \mathrm{E}-01$ & 0.32 & $3.46 \mathrm{E}-01$ & $\mathrm{n} / \mathrm{a}$ & $\begin{array}{r}\text { Propeptide domain containing protein } \\
\text { [U6PXI5] }\end{array}$ & $\mathrm{n} / \mathrm{a}$ & pep-2 & AJ577754.1 $\frac{1}{c}$ \\
\hline & HCOI01993500 & 13499.65 & 0.34 & $2.65 \mathrm{E}-01$ & 0.35 & $3.06 \mathrm{E}-01$ & $\mathrm{n} / \mathrm{a}$ & $\begin{array}{r}\text { Propeptide and Peptidase A1 domain } \\
\text { containing protein [U6PQD5] }\end{array}$ & $\mathrm{n} / \mathrm{a}$ & pep-1 & AF079402.1@ \\
\hline & HCOI00348800 & 8859.39 & 0.47 & $1.56 \mathrm{E}-02$ & 0.51 & $1.14 \mathrm{E}-01$ & $\mathrm{n} / \mathrm{a}$ & $\begin{array}{r}\text { Peptidase M13 domain containing protein } \\
\text { [U6NMI3] }\end{array}$ & $\mathrm{n} / \mathrm{a}$ & mep-2 & AF080117.1 \\
\hline & HCOI01333400 & 9325.90 & 0.59 & 3.88E-02 & 0.62 & $1.64 \mathrm{E}-01$ & $\mathrm{n} / \mathrm{a}$ & $\begin{array}{r}\text { Peptidase M13 domain containing protein } \\
\text { [U6PHP6] }\end{array}$ & $\begin{array}{r}\text { nep-9, nep- } \\
20\end{array}$ & mep-3 & $\mathrm{AF} 080172.1$ \\
\hline & HCOI02032800 & 2207.13 & 0.71 & $1.25 \mathrm{E}-02$ & 0.90 & $5.97 \mathrm{E}-02$ & $\mathrm{n} / \mathrm{a}$ & $\begin{array}{r}\text { Peptidase M1 domain containing protein } \\
\text { [U6PYE0] }\end{array}$ & T07F10.1 & h11 & FJ481146.1 \\
\hline & HCOI00308300 & 18250.90 & 0.73 & 4.82E-04 & 0.78 & $5.85 \mathrm{E}-02$ & $\mathrm{n} / \mathrm{a}$ & $\begin{array}{l}\text { Peptidase M13 domain containing protein } \\
\text { [U6NME0] }\end{array}$ & & mep-1 & AF102130.1 \\
\hline & HCOI00631000 & 5690.45 & 0.77 & 2.40E-04 & 0.81 & $5.97 \mathrm{E}-02$ & $\mathrm{n} / \mathrm{a}$ & & & mep-4 & AF132519.1 \\
\hline \multirow{5}{*}{ 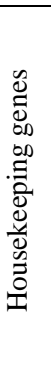 } & HCOI00909100 & 5753.25 & -0.41 & $5.29 \mathrm{E}-01$ & -0.60 & $3.93 \mathrm{E}-01$ & $\mathrm{n} / \mathrm{a}$ & $\begin{array}{l}\text { Nematode fatty acid retinoid binding } \\
\text { domain containing protein [U6NYW0] }\end{array}$ & $\mathrm{n} / \mathrm{a}$ & far & CDJ86885.1 \\
\hline & HCOI00117100 & 1379.12 & 0.08 & $9.64 \mathrm{E}-01$ & 0.07 & $7.96 \mathrm{E}-01$ & $\mathrm{n} / \mathrm{a}$ & Superoxide dismutase [Cu-Zn] [U6NGP5] & $\mathrm{n} / \mathrm{a}$ & sod & CDJ80830.1 \\
\hline & HCOI01760600 & 24868.64 & 0.08 & $8.59 \mathrm{E}-01$ & 0.08 & 7.92E-01 & $\mathrm{n} / \mathrm{a}$ & $\begin{array}{r}\text { Glyceraldehyde-3-phosphate } \\
\text { dehydrogenase (inferred by orthology to a } \\
\text { human protein) } \\
\text { [Source:UniProtKB;Acc:P04406] }\end{array}$ & $\mathrm{n} / \mathrm{a}$ & gpd & CDJ92718.1 \\
\hline & HCOI01743600 & 194.02 & 0.13 & $9.13 \mathrm{E}-01$ & 0.14 & $7.28 \mathrm{E}-01$ & $\mathrm{n} / \mathrm{a}$ & $\begin{array}{r}\text { RNA recognition motif domain containing } \\
\text { protein [U6NLP1] }\end{array}$ & $\mathrm{n} / \mathrm{a}$ & ncbp & CDJ82645.1 \\
\hline & HCOI01464300 & 974.31 & 0.32 & $3.24 \mathrm{E}-01$ & 0.35 & $3.06 \mathrm{E}-01$ & $\mathrm{n} / \mathrm{a}$ & DNA-directed RNA polymerase [U6PFA6] & $\mathrm{n} / \mathrm{a}$ & ama & CDJ91461.1 \\
\hline
\end{tabular}



adjusted for multiple testing; ${ }^{\mathrm{d}}:$ correlation between transcript expression level and Faecal Egg Count at 29 day post-infection. 


\section{Supporting information}

503 Supplementary Figure1. Principal component analysis (PCA) of transcript counts measured in worms collected in vaccinated or control sheep

PCA is a dimensionality reduction method that makes use of transcript counts to define a new set of unrelated components. Coordinates of every pool of worms considered for analysis is plotted against first two components and correlate with similarities between pools. The first PCA axis explains $36 \%$ of total variance and relates to differences between the two considered experimental groups, i.e. worms exposed to the vaccine response (V) or the control group (C).

Supplementary Figure2. Number of differentially expressed genes found by each of the two implemented methods

514 Total number of significantly differentially expressed genes found by at least one of the two methods (DESeq2, VOOM, or both (intersecting)) are plotted according to their regulation pattern, i.e up or down-regulated in the vaccine survivors, to their estimated fold change, i.e. $\log 2 \mathrm{FC}>2,1$ or 0 .

519 Supplementary Table 1. Faecal egg count and worm volumes recovered at necropsy and 520 RNA-seq library details

521 Supplementary Table 2. List of primer sequences used for qPCR validation

522 Supplementary Table 3. Complete list of differentially expressed genes

523 Supplementary Table 4. Transcript count of the six most differentially expressed genes in 524 pools of male worms from each sheep

525 Supplementary Table 5. Significantly enriched GO terms associated with the differentially 526 expressed genes 
bioRxiv preprint doi: https://doi org/10.1101/144980; this version posted October 24, 2017. The copyright holder for this preprint (which was not certified by peer review) is the author/funder, who has granted bioRxiv a license to display the preprint in perpetuity. It is made available under aCC-BY-NC 4.0 International license.

527 
A

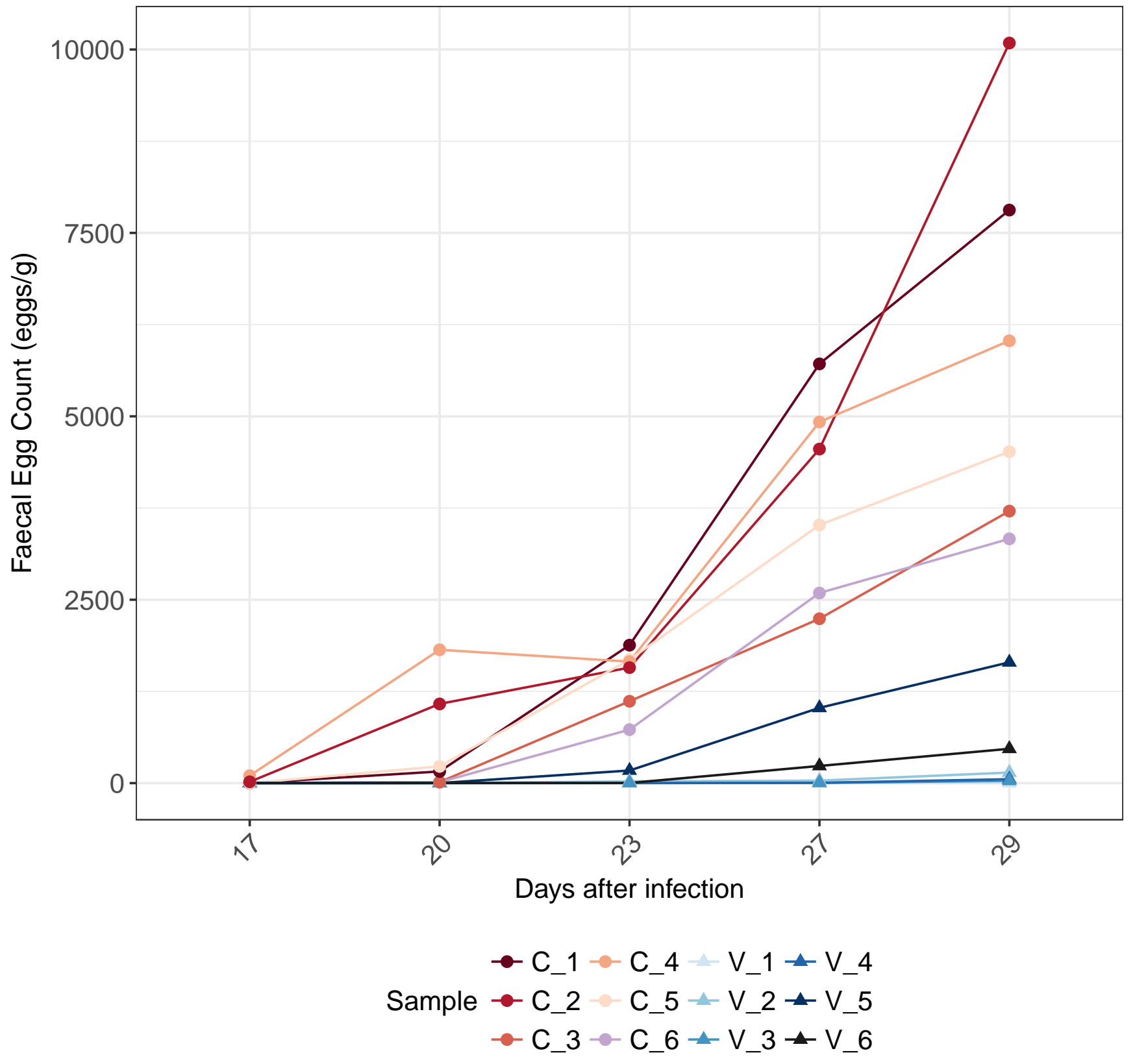




\section{B}

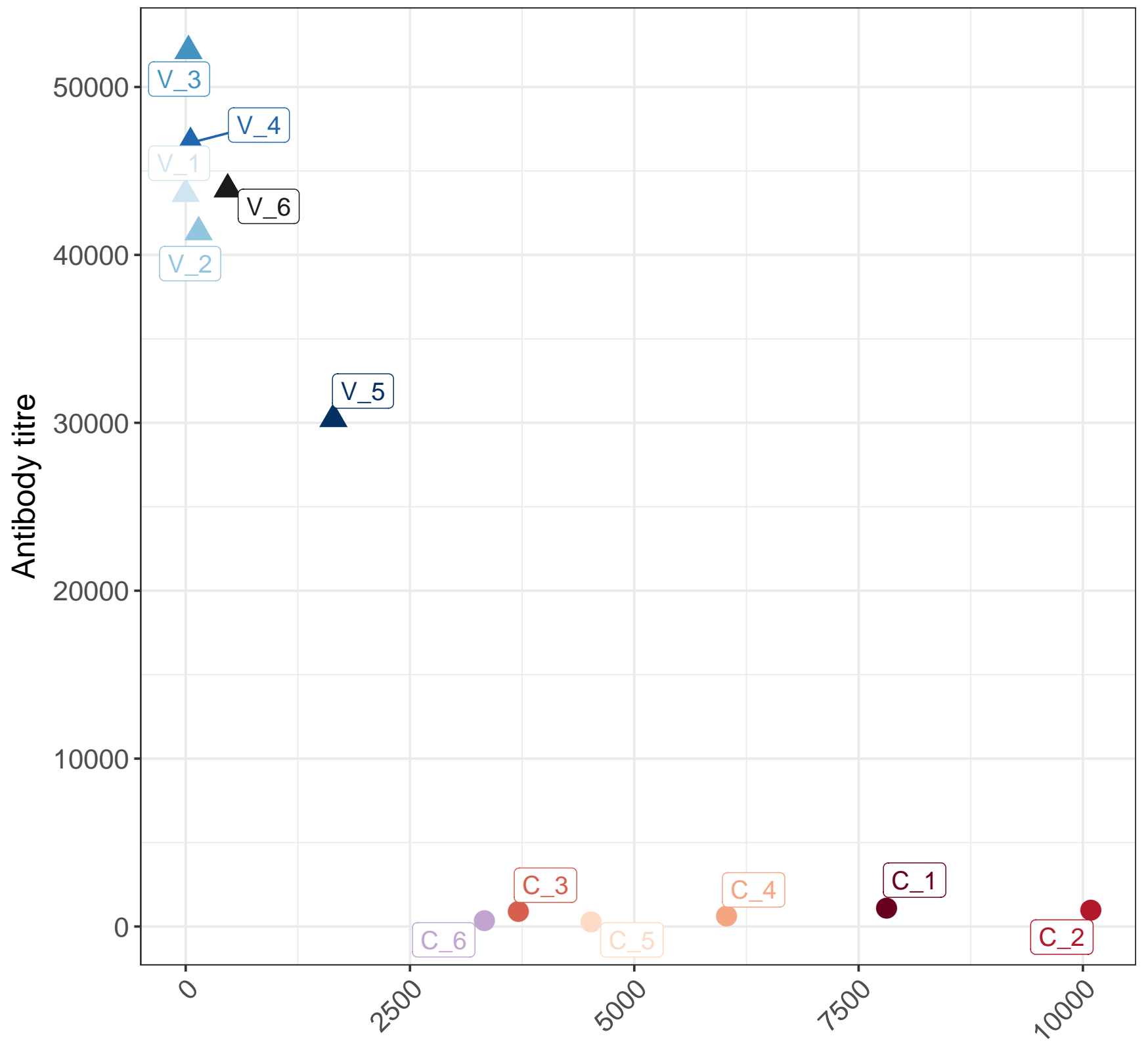

Faecal Egg Count at necropsy (eggs/g) 
A

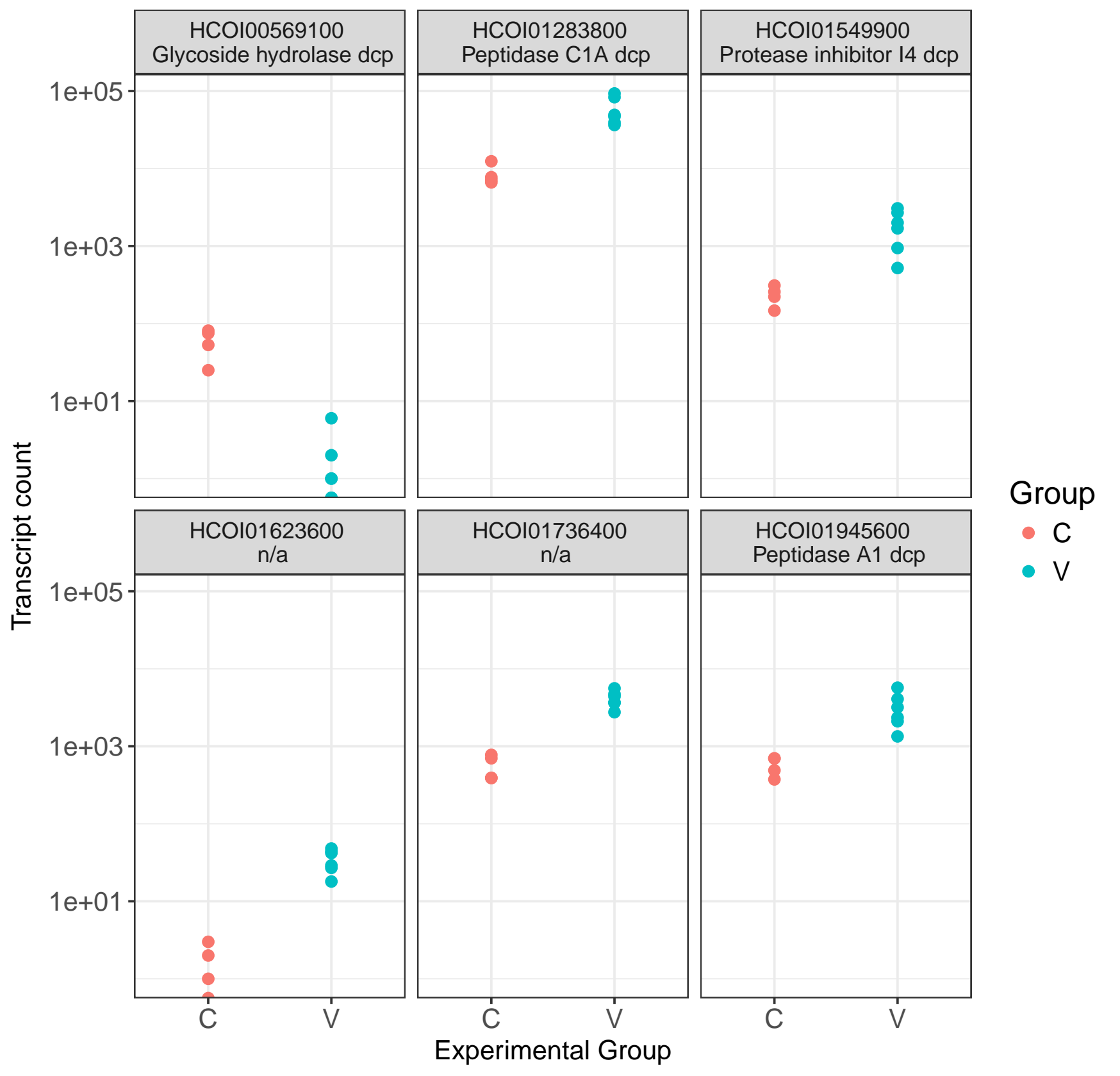




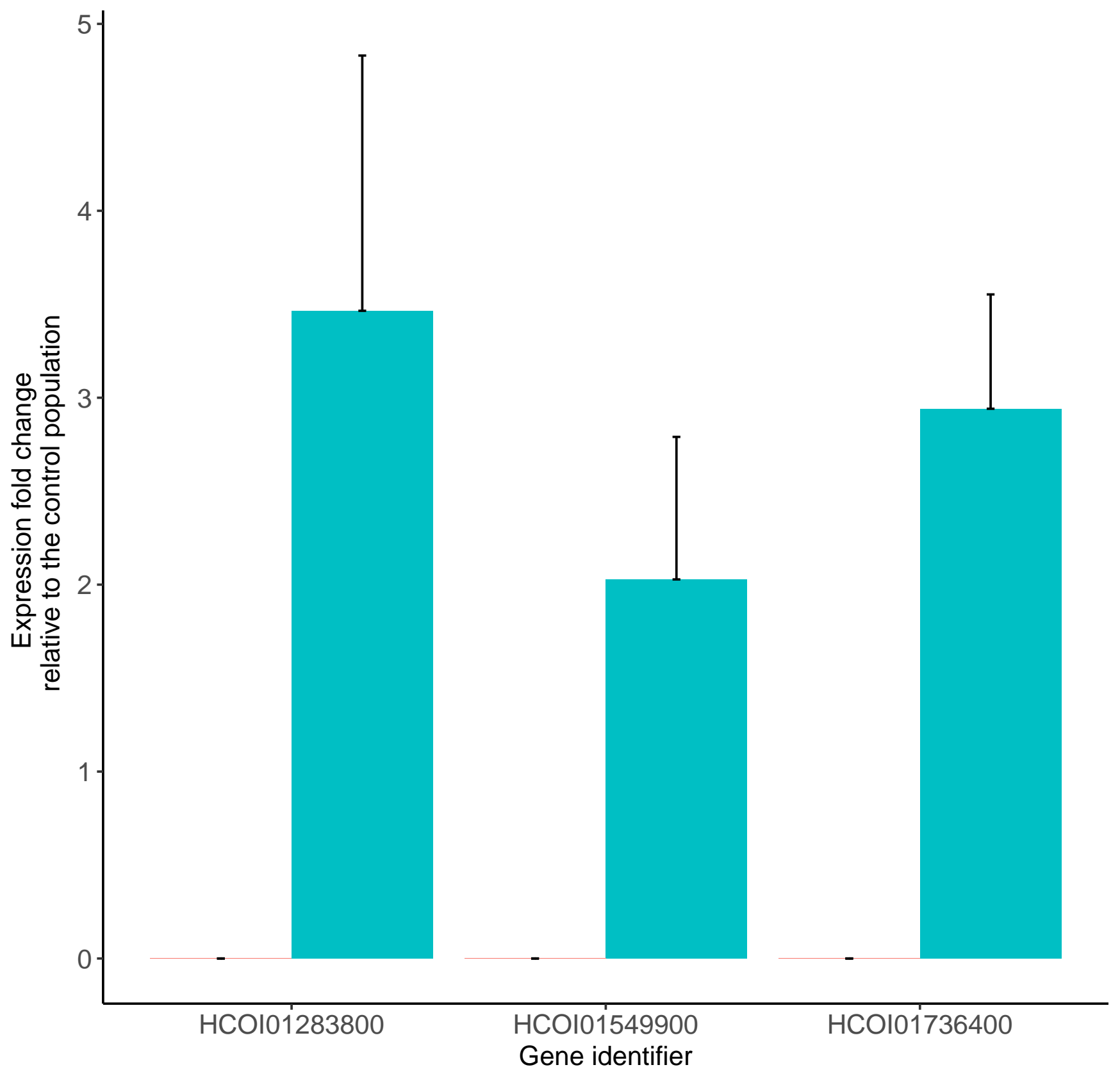



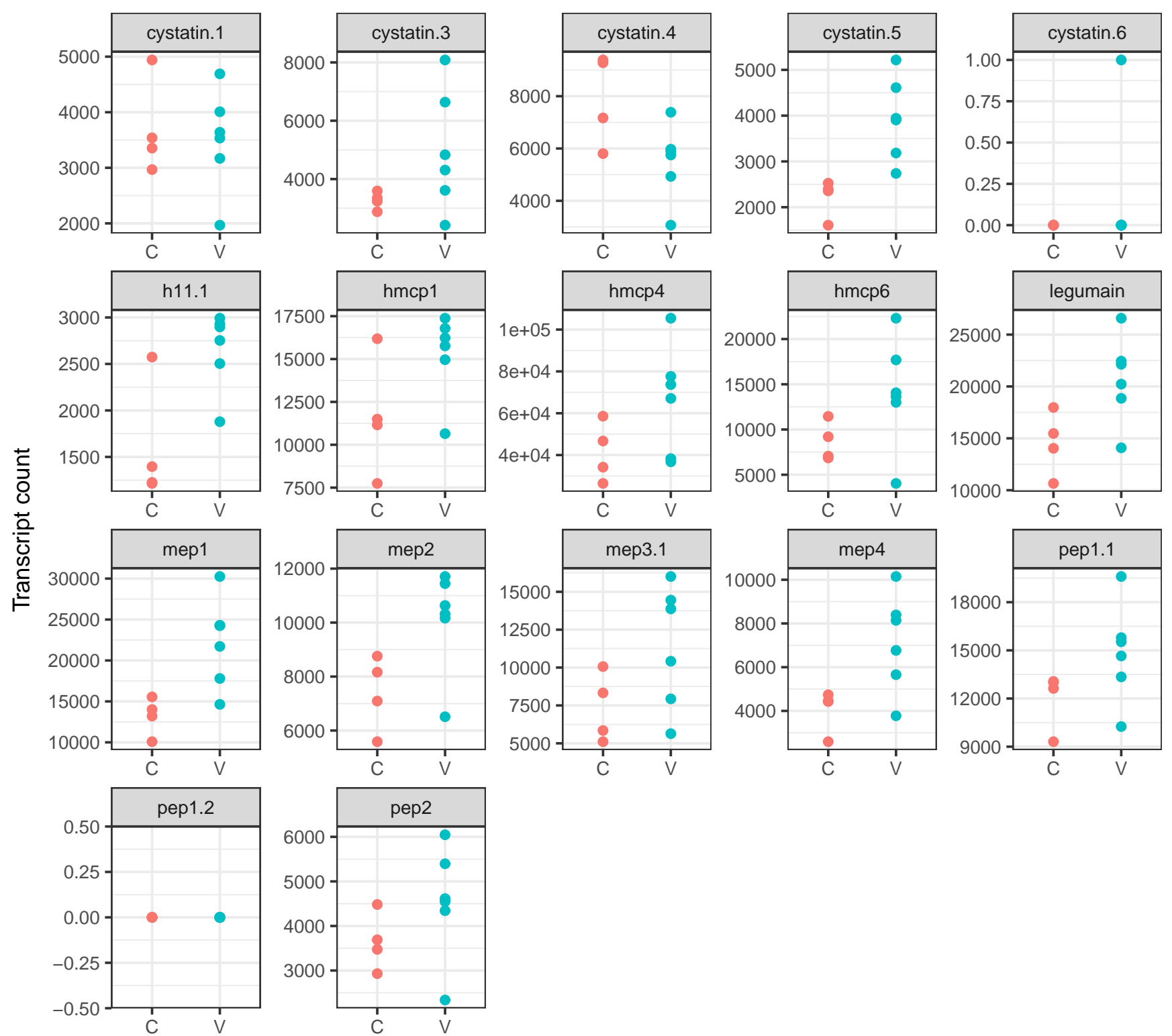

Experimental Group

Group - C V 